\title{
COMPUTER-AIDED DESIGN METHOD OF WARP-KNITTED JACQUARD SPACER FABRICS
}

\author{
Xinxin Li, Gaoming Jiang*, Pibo Ma* \\ Engineering Research Center for Knitting Technology, Ministry of Education, \\ Jiangnan University, Jiangsu, Wuxi, 214122, China \\ *E-mail: Jiang G. jiang@526.cn Ma P. mapibo@jiangnan.edu.cn
}

\begin{abstract}
:
Based on a further study on knitting and jacquard principles, this paper presents a mathematical design model to make computer-aided design of warp-knitted jacquard spacer fabrics more efficient. The mathematical model with matrix method employs three essential elements of chain notation, threading and Jacquard designing. With this model, the processing to design warp-knitted jacquard spacer fabrics with CAD software is also introduced. In this study, the sports shoes which have separated functional areas according to the feet structure and characteristics of movement are analysed. The results show the different patterns on Jacquard spacer fabrics that are seamlessly stitched with jacquard technics. The computer-aided design method of warp-knitted jacquard spacer fabrics is efficient and simple.
\end{abstract}

\section{Keywords:}

warp knitting; jacquard; spacer fabrics; computer-aided design method

\section{Introduction}

With the application of Piezo-Jacquard mechanism in warpknitting machine, warp-knitted jacquard spacer fabrics with rich and changeable jacquard effects have recently become popular. These fabrics are mostly applied on sports shoes, mattress ticking, garments, internal decorations in automobiles, seating furniture and mouldable spacer. The fabrics are produced on double needle bar Raschel machine with Piezo-Jacquard system, which means that technics design must be aided by a computer software. Computer-aided design method tends to be the leading edge of warp-knitting development. However, the existing studies on computer-aided design method of warpknitted fabrics are mainly concentrated on multibar Raschel lace and jacquard curtains [1-2]. Likewise, researches on warpknitted spacer fabrics are mainly focused on product design, application, knitting technique, physical property and mechanical performance [3-6]. Although there are many studies on warpknitted jacquard fabrics and spacer fabrics, there are few researches on jacquard spacer fabrics, especially their design aided by computer software. Therefore, this paper presents a method for the computer-aided design of warp-knitted jacquard spacer fabrics by mathematical matrix and visual $\mathrm{C}++$ programming language based on a further analysis of warp knitting and jacquard principles. This computer-aided design method makes it more convenient and efficient to design and promote new warp-knitted jacquard spacer products and offers extended patterning possibilities and increased productivity.

\section{Knitting technics}

\section{$\underline{2.1 \mathrm{Knitting} \text { principle }}$}

Warp-knitted spacer fabric is a three-dimensional fabric, as shown in Figure 1 [6], comprising a top surface, a bottom side and a pile-yarn layer. This three-dimensional fabric is knitted on Raschel machines equipped with two individual latch needle beds and one or two Piezo-Jacquard guide bars. The PiezoJacquard guide bars are constructed of piezoelectric elements to characterize the pattern surface by knitting floral patterns, logos, characters as well as versatile net variations. Both the pattern side and bottom side are knitted on one single bed by more than one guide bars while the pile-yarn layer is formed on two beds with lapping movements $1-0-1-2 / 2-3-2-1 / /$ or $1-0-$ $2-3 / 4-5-3-2 / /$ to link pattern and bottom side by loops. With this method, the thickness between pattern side and bottom side can be adjusted from $2 \mathrm{~mm}$ to $8 \mathrm{~mm}$ by optionally tuning knockover comb bars distance.

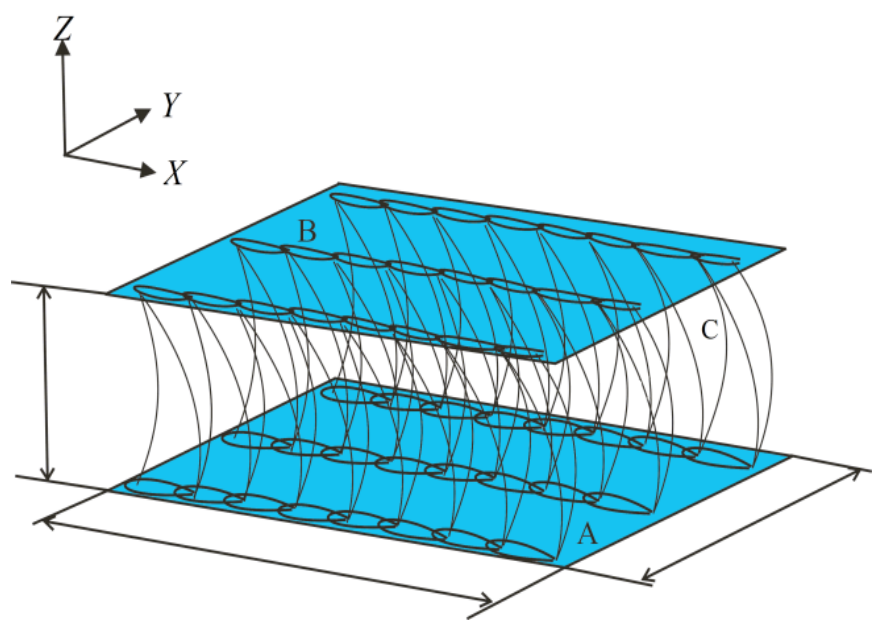

Figure 1. 3-Dstructure of warp-knitted spacer fabrics (X-warp direction)

\subsection{Jacquard working principle}

Piezo-Jacquard guide bars, two separate jacquard bars in half gauge, are equipped on back needle bed of the double 
needle bar Raschel machine with Piezo-Jacquard system. These jacquard bars are aimed at knitting versatile net variations, characters and logos on spacer fabrics with basic lapping movement 1-1-1-0/1-1-1-2//. Each individual jacquard guide needle is a single piezoelement and it is independently controlled by Piezo-Jacquard system. The piezoelectric element is made up two piezo ceramics isolated with a layer of glass fibre. Piezo ceramics are characterized to deform by itself and displace the needle to the left or right hand side of the jacquard guide when a voltage of $200 \mathrm{~V}$ is alternatingly set on each side of the piezo element with switching. Therefore, Piezo-Jacquard system enables the jacquard guide needle to be displaced both overlapping and underlapping, forming two displacements in one course and improving the feasibility of varied effects. One course knitted on the double needle bed Raschel machine consists of two loops and one is formed on the front latch needle bed and the other on the back latch needle bed, as shown in Figure 2. When the jacquard guide needle works on the front needle bed, it is displaced on the right side after receiving an electric signal $H$ from control system to knit plain stitches while displaced to the left side after receiving an electric signal $T$ to knit thick and tight stitches or loose meshes [1] on the back needle bed.

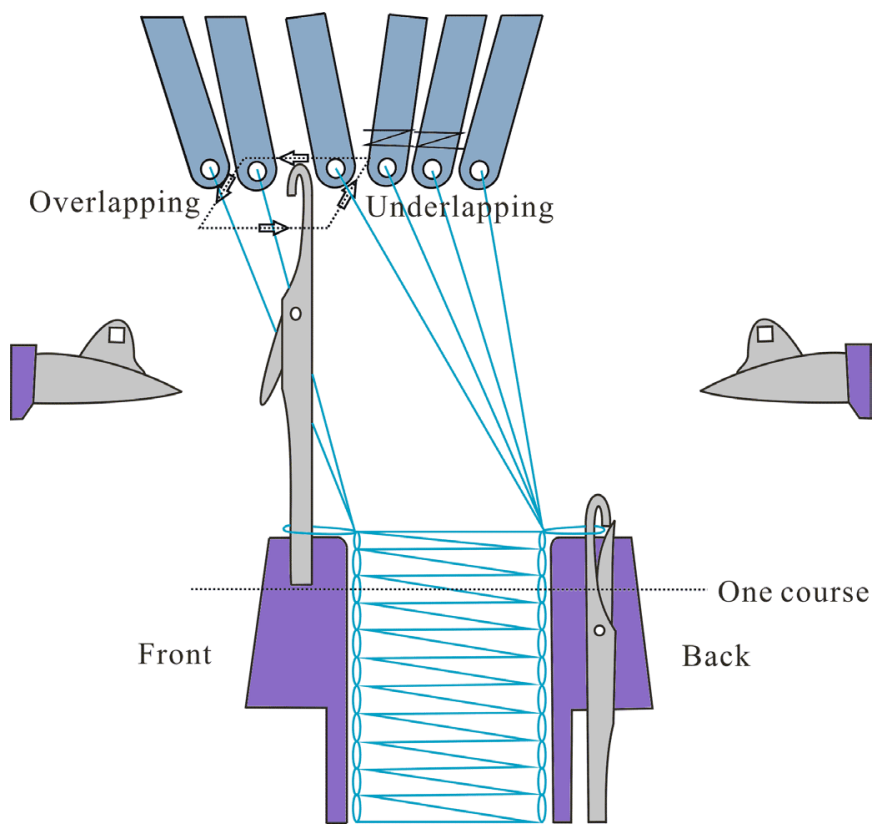

Figure 2. Knitting process of double needle bar Raschel machine
For most of the computer-aided design systems for warp knitting fabrics, jacquard grid is the basic unit for jacquard design and one grid represents two courses. This paper assumes the first course as the odd one and the second course as the even one. Thus there are eight signals of displacement in one grid. Signals 1 and 2 represent the odd course in front bed, while signals 3 and 4 represent the odd course in back bed. For the even course, lapping movement in front bed is controlled by signals 5 and 6 , whereas the lapping movement in back bed is controlled by signals 7 and 8 . The jacquard surface knitted on the back bed is usually assumed to be knitted on the front bed when designed on CAD software [1], in which case the jacquard guide needle is displaced to the left side with signal $T$ and to the right side with signal $H$ on the basic chain notation 1-1-1-0/1-1-1-2//. The displacement rule of jacquard needle and its corresponding jacquard colour when designing on CAD software are shown in Table 1.

\section{Design model of jacquard spacer fabric}

The most important link of producing warp-knitted jacquard spacer fabrics is technics design and it is attached with three key elements: lapping movement, threading cycle and jacquard design. Therefore, three mathematical models are needed for achieving the computer-aided design of jacquard spacer fabrics.

\subsection{Lapping design model}

Pattern driving devices, such as pattern disks, pattern drums and electronic guide bar drives, are equipped on the right side of the double needle bar Raschel machine with Piezo-Jacquard system, so clearances between two latch needles are always numbered with $0,1,2,3$, etc. from right to left. These numbers are used to show lapping structures of guide bars when they are driven by pattern drives. Different from common warp-knitted fabrics whose chain notation for each course only has two natural numbers, the chain notation of jacquard spacer fabrics for each course has four numbers and they are connected with short lines [7], as shown in Tab. 1. Mathematical model of lapping design adopts a three-dimensional matrix $F_{L M 4}(i, j, 4)$ hence, where: $i \in\{1,2,3,4,5, \ldots L\}, j \in\{1,2,3,4,5, \ldots M\}$; $L$ is the total number of guide bars; $M$ is the number of courses in one

Table 1. Jacquard principle on back needle bed

\begin{tabular}{|c|c|c|c|c|c|}
\hline Displaced course & Signals & Chain notation & Lapping & Colour & Effect \\
\hline Even & $\begin{array}{l}\mathrm{HHHH}- \\
\text { HHTT }\end{array}$ & $\begin{array}{l}2-2-1-0 / \\
1-1-2-3 / /\end{array}$ & & Red & Thick \\
\hline None & $\begin{array}{l}\mathrm{HHHH}- \\
\mathrm{HHHH}\end{array}$ & $\begin{array}{l}1-1-1-0 / \\
1-1-1-2 / /\end{array}$ & $3=b_{0^{\mathrm{B}}}^{\mathrm{F}}$ & Green & Thin \\
\hline Both & $\begin{array}{l}\text { HHTT- } \\
\text { HHTT }\end{array}$ & $\begin{array}{l}2-2-2-1 / \\
2-2-2-3 / /\end{array}$ & ${ }_{3} \cdot{ }_{2} \rho_{1}{ }^{\text {в }}$ & Blue & Thin \\
\hline Odd & $\begin{array}{l}\text { HHTT- } \\
\text { HHHH }\end{array}$ & $\begin{array}{l}2-2-2-1 / \\
1-1-1-2 / /\end{array}$ & - & White & Mesh \\
\hline
\end{tabular}


pattern repeat. In this paper, $f(i, j, 1)$ and $f(i, j, 2)$ are separately taken as the numbers where lapping path of bar No.i starts and ends when knitting course No. $j$ is in the front bed, likewise, $f(i, j, 3)$ and $f(i, j, 4)$ as the numbers when knitting is in the back bed. The chain notation of bar No. $i$ when knitting course No. $j$ is described with a one-dimensional matrix $[f(i, j, 1), f(i, j, 2), f(i, j, 3)$, $f(i, j, 4)]$, and the mathematical model of chain notation for all guide bars in one pattern repeat is shown in formula (1).

$F_{L}{ }_{4}=\left[\begin{array}{cccc}f_{i 11} & f_{i 12} & f_{i 13} & f_{i 14} \\ \vdots & \vdots & \vdots & \vdots \\ f_{i j 1} & f_{i j 2} & f_{i j 3} & f_{i j 4} \\ \vdots & \vdots & \vdots & \vdots \\ f_{i M 1} & f_{i M 2} & f_{i M 3} & f_{i M 4}\end{array}\right]$

When the guide bar No. $i$ knits course No. $j$, the overlapping movement in the front bed is described as $F L(i, j)$ and the underlapping movement as $F L^{\prime}(i, j)$. However, in the back needle bed, the overlapping movement is shown with $B L(i, j)$ and the underlapping movement is $B L^{\prime}(i, j)$. Therefore, $F L(i, j)=f(i, j, 2)$ $f(i, j, 1), \quad B L(i, j)=f(i, j, 4)-f(i, j, 3), \quad B L^{\prime}(i, j)=f(i, j, 3)-f(i, j, 2) \quad$ when $j=1$, $F L^{\prime}(i, j)=f(i, j, 1)-f(i, J, 4)$ when $j \geq 2$ and $F L^{\prime}(i, j)=f(i, j, 1)-f(i, j-1,4)$. Lapping movement on the front needle bed is separately decided by the value of $F L(i, j), F L^{\prime}(i, j)$ and the product of the two arrays, as shown in Table 2. Lapping movement on the back needle bed is determined by the same method. For example, guide bar NO.4 for RDPJ7/1 knits pile-yarn layer with lapping movements 1-0-1-2/2-3-2-1// and its mathematical model of chain notation can be described as

$F_{\text {LM } 4}=\left[\begin{array}{llll}f(4,1,1) & f(4,1,2) & f(4,1,3) & f(4,1,4) \\ f(4,2,1) & f(4,2,2) & f(4,2,3) & f(4,2,4)\end{array}\right]=\left[\begin{array}{llll}1 & 0 & 1 & 2 \\ 2 & 3 & 2 & 1\end{array}\right]$,

where:

$B L(4,1)=f(4,1,4)-f(4,1,3)=1>0, \quad B L^{\prime}(4,1)=f(4,1,3)-f(4,1,2)=0$, $F L^{\prime}(4,1)=f(4,1,1)-f(4,2,4)=0, \quad F L(4,2)=f(4,2,2)-f(4,2,1)=1>0$, $B L(4,2)=f(4,2,4)-f(4,2,3)=-1<0, \quad B L^{\prime}(4,2)=f(4,2,3)-f(4,2,2)=-1<0$, $F^{\prime}(4,2)=f(4,2,1)-f(4,1,4)=0$.

Table 2. Lapping movement of guide bars on front needle bed

\begin{tabular}{|c|c|c|}
\hline Bed & Value & Lapping movement \\
\hline \multirow{11}{*}{ Front } & $F L(i, j)<0$ & Overlapping left to right \\
\hline & $F L(i, j)=0$ & inlay \\
\hline & $F L(i, j)>0$ & Overlapping right to left \\
\hline & $F L^{\prime}(i, j)<0$ & Underlapping left to right \\
\hline & $F L^{\prime}(i, j)=0$ & float \\
\hline & $F L^{\prime}(i, j)>0$ & Underlapping right to left \\
\hline & $F L(i, j)=1$ & Common lapping \\
\hline & $F L(i, j)=2$ & Koper stitch \\
\hline & $F L(i, j) \times F L^{\prime}(i, j)>0$ & Open stitch \\
\hline & $F L(i, j) \times F L^{\prime}(i, j)=0$ & Inlay or float \\
\hline & $F L(i, j) \times F L^{\prime}(i, j)>0$ & Closed stitch \\
\hline
\end{tabular}

\subsection{Threading design model}

Threading situation of guide needle bars is shown by threading cycle, and yarns with different threading situations, materials, colours or diameters can be knitted to provide different fabric effects of patterns. To show the threading situation in computeraided design software, a two-dimensional matrix $T L C=t(i, c)$ is employed in this paper, as shown in formula (2), where: $i \in\{1,2$, $3,4,5, \ldots L\}, C \in\{1,2,3,4,5, \ldots C\} ; \mathrm{L}$ is the number of guide bars and $C$ is the number of wales in one pattern repeat, namely width.

$$
T_{L C}=\left[\begin{array}{cccccc}
t_{11} & t_{12} & \ldots & t_{1 c} & \ldots & t_{1 C} \\
\vdots & \vdots & \vdots & \vdots & & \vdots \\
t_{i 1} & t_{i 2} & \ldots & t_{i c} & \ldots & t_{i C} \\
\vdots & \vdots & \vdots & \vdots & & \vdots \\
t_{L 1} & t_{L 2} & \ldots & t_{L C} & \ldots & t_{L C}
\end{array}\right]
$$

The value of $t(i, c)$ can be represented with letters ranging from $A$ to $Z$ to show one specific kind of yarn, namely tic $\in\{A, B, C, D$, $E, \ldots Z\}$. However, when there is no yarn threaded, the value of $t(i, c)$ is represented with a symbol *. For example, the threading situation of matrix $\left[\begin{array}{llll}A & * & * & A \\ B & B & B & *\end{array}\right]$ is shown in Figure 3 .

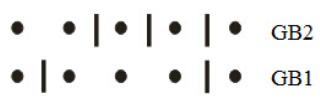

Figure 3. Threading design

\subsection{Jacquard design model}

Jacquard design is the key for designing warp-knitted jacquard spacer fabrics, and jacquard grids are filled with different colours to represent different lapping movements [8]. The paper uses a two-dimensional matrix $J C N=j(c, n)$ to show jacquard pattern information in one repeat, as shown in formula (3), where: $c \in\{1,2,3,4,5, \ldots C\}, n \in\{1,2,3,4,5, \ldots N\} ; C$ is the number of wales in one repeat, namely width; $N$ is the vertical number of grids, namely half of the height owing to two courses in one grid. Generally, the value of $j(c, n)$ equals to $1,4,8$ or 12 to respectively mean thick effect, thin effect, thin effect and mesh. For example, if matrix

$J=\left[\begin{array}{lll}j(1,1) & j(1,2) & j(1,3) \\ j(2,1) & j(2,2) & j(2,3) \\ j(3,1) & j(3,2) & j(3,3)\end{array}\right]=\left[\begin{array}{ccc}1 & 4 & 12 \\ 8 & 1 & 4 \\ 12 & 8 & 1\end{array}\right]$,

then the matrix of signals controlling jacquard elements equals to

$$
\begin{gathered}
{\left[\begin{array}{ccc}
\mathrm{H} & \mathrm{H} & \mathrm{T} \\
\mathrm{T} & \mathrm{H} & \mathrm{H} \\
\mathrm{T} & \mathrm{H} & \mathrm{H} \\
\mathrm{T} & \mathrm{T} & \mathrm{H} \\
\mathrm{T} & \mathrm{T} & \mathrm{H} \\
\mathrm{H} & \mathrm{T} & \mathrm{T}
\end{array}\right]} \\
I_{C N}
\end{gathered}
$$




\section{Design of jacquard spacer fabrics with CAD}

The most competitive merit of warp-knitted jacquard spacer fabrics is seamlessly stitching technic because this technic transition areas between different jacquard effects are favourable, such as Adidas Climachill sports shoe shown in Figure 4. According to characteristics of feet and its movement, the sports shoe is separated into six functional areas, and the upper area marked with $A$ and the area marked with $F$ are large meshes for good air permeability, whereas the toe cap area marked with $C$ and the heel area marked with $B$ are small meshes. Comparatively, collar area marked with $E$ and toe cap area marked with $D$ are dense and thick stitches that provide good strength. These six areas can be seamlessly stitched with jacquard technics, reducing seaming operation.

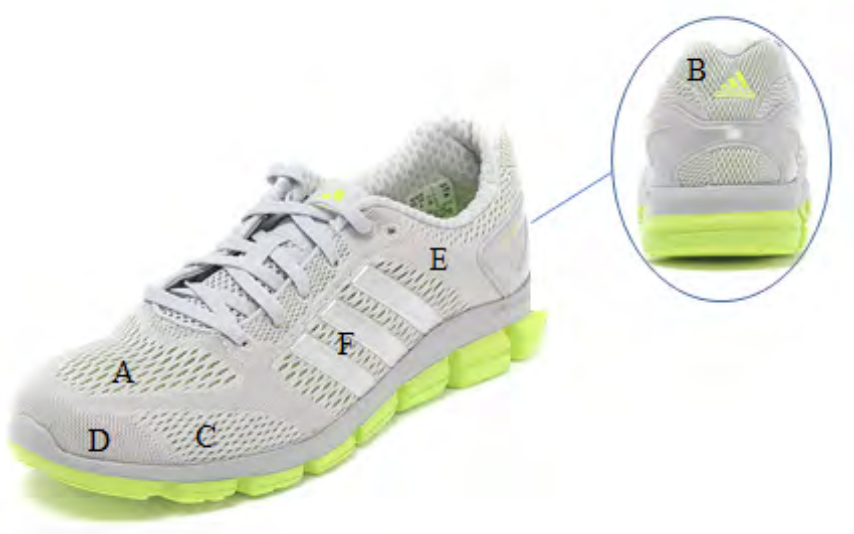

Figure 4. Adidas Climachill sports shoes

When wearers are doing physical exercises, heat and sweat are produced by feet and released out of shoes in level and vertical directions, which is shown in Figure 5. To offer a favourable climatic regulation with good air permeability, welldirected moisture and heat dissipation, warp-knitted jacquard spacer fabrics for shoes are usually characterized by the meshes of various sizes knitted by Piezo-Jacquard technics. The following part focuses on technics design of jacquard spacer shoes shown in Figure 4 and its functional areas are separated as shown in Figure 6.

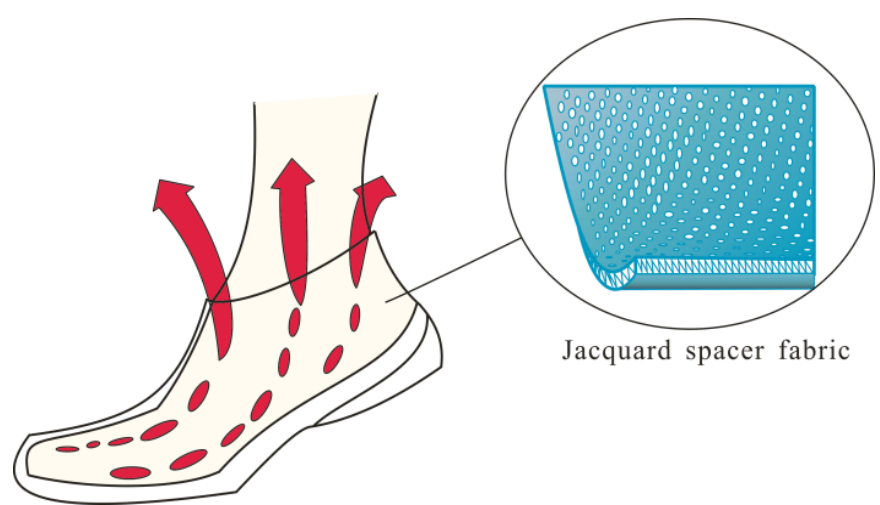

Figure 5. Heat dispersion of sports shoes

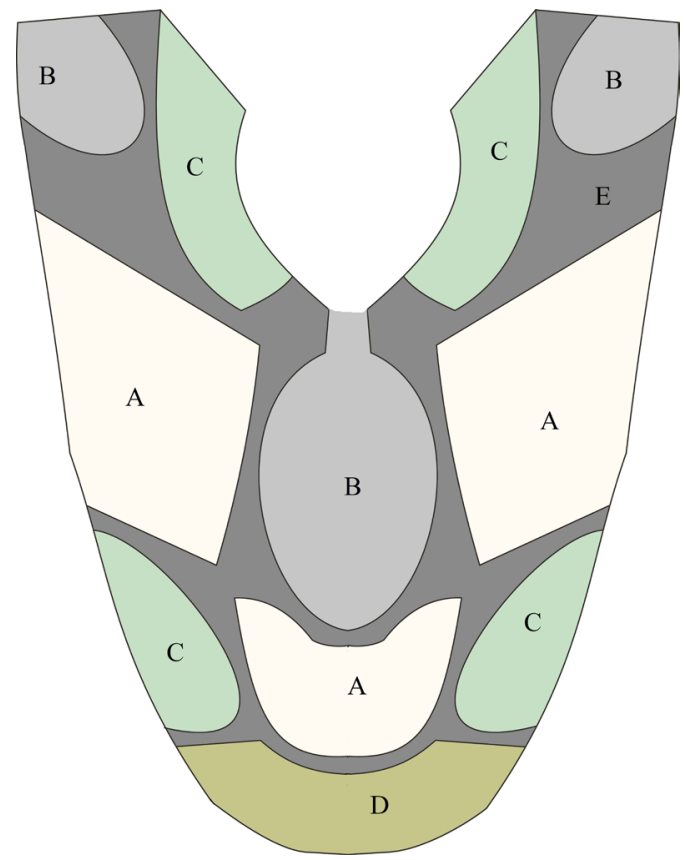

Figure 6. Separated functional areas

\subsection{Technical data design}

Machine: RDPJ7/1EL; working width: $350 \mathrm{~cm}$; gauge: E24; working speed: $300 \mathrm{rad} / \mathrm{min}$; finished WPC: 10.7; finished CPC: 16.3; production: $11 \mathrm{~m} / \mathrm{h}$; gram weight: $293 \mathrm{~g} / \mathrm{m}^{2}$.

\subsection{Lapping movement and threading cycle}

GB2: $1-0-1-1 / 1-2-1-1 / 1-0-2-2 / 4-5-4-4 / 4-3-4-4 / 4-5-3-3 / /$ one in one out, 167 dtex hemi light polyester;

GB3: 4-5-4-4/4-3-4-4/4-5-3-3/1-0-1-1/1-2-1-1/1-0-2-2// one out one in, 167 dtex hemi light polyester;

GB4: 2-1-1-0/1-2-2-3// fully threaded, 33 dtex hemi light polyester;

JB5.1: 1-1-1-0/1-1-1-2// one out one in, 156 dtex hemi light nylon;

JB5.2: 1-1-1-0/1-1-1-2// one in one out, 156 dtex hemi light nylon;

GB6: 1-1-1-0/0-0-0-1// fully threaded, 78 dtex hemi light nylon; GB7: 1-1-0-0/0-0-1-1// fully threaded, 78 dtex hemi light nylon.

With the design models built above, lapping movements of all guide bars are automatically drawn after typing in chain notations on computer-aided design software with visual $\mathrm{C}++$ as programming language (shown in Figure 7). The design interface can also be selectively switched from front bed, back bed and pile-yarn layer to make designing more convenient and efficient.

\subsection{Jacquard design}

For the sports shoe shown in Figure 5, there are six functional areas designed with appropriate jacquard effects (listed in Table 3) and the final jacquard pattern design is shown in Figure 8 with logo Adidas drawn directly by jacquard text design function. 


\begin{tabular}{|l|l|l|l|l|l|l|l|l|}
\hline & GB1 & GB2 & GB3 & GB4 & JB5.1 & JB5.2 & GB6 & GB7 \\
\hline 1 & & $1-0-1-1$ & $4-5-4-4$ & $2-1-1-0$ & $1-1-1-0$ & $1-1-1-0$ & $1-1-1-0$ & $1-1-0-0$ \\
\hline 2 & $1-2-1-1$ & $4-3-4-4$ & $1-2-2-3$ & $1-1-1-2$ & $1-1-1-2$ & $0-0-0-1$ & $0-0-1-1$ \\
\hline 3 & & $1-0-2-2$ & $4-5-3-3$ & & & & & \\
\hline 4 & $4-5-4-4$ & $1-0-1-1$ & & & & & \\
\hline 5 & $4-3-4-4$ & $1-2-1-1$ & & & & & \\
\hline 6 & $4-5-3-3$ & $1-0-2-2$ & & & & & \\
\hline
\end{tabular}

(a)

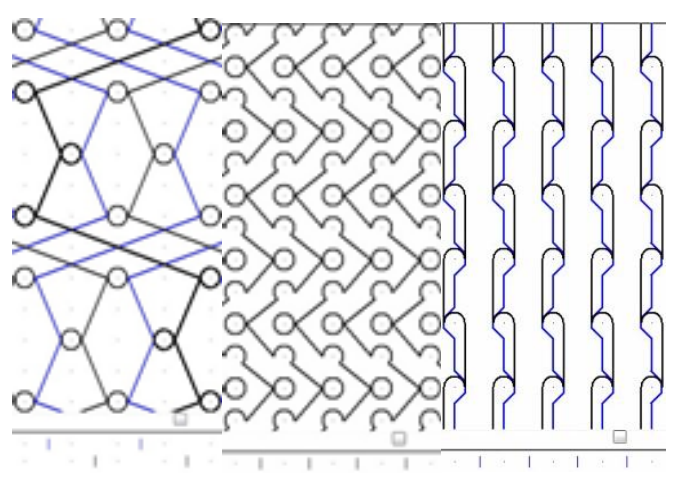

(b)

(c) (d)

Figure 7. Lapping design. Chain notation (a), Lapping movements of front needle bed (b), front and back needle beds (c) and back needle bed (d).

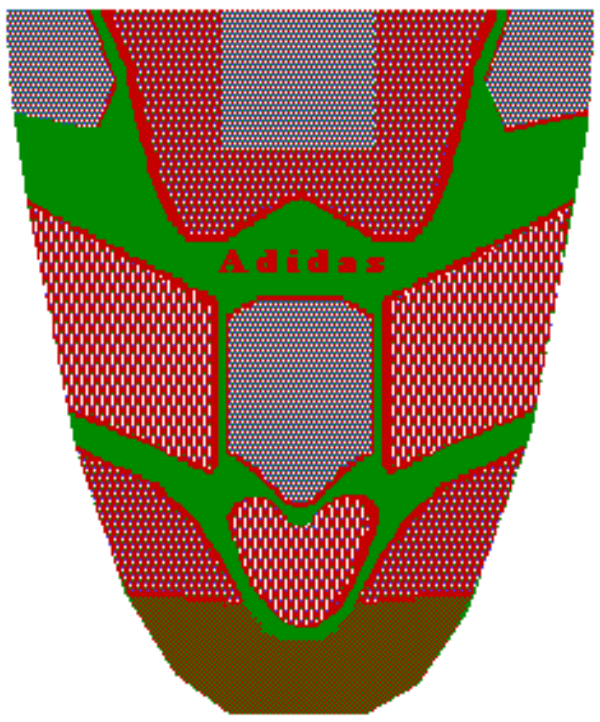

Figure 8. Jacquard design
After designing the lapping movement, yarns, threading cycle and jacquard pattern, the gram weight and production can be automatically calculated by the CAD software, thus decreasing the design time.

\section{Conclusion}

1) Mathematical models of lapping movement, threading cycle and jacquard pattern design are given in this paper based on a study on knitting principles and jacquard technics of warpknitted jacquard spacer fabrics, thus improving CAD design functions for the fabrics. The mathematical models are proved accurate by taking the technics design of jacquard spacer sports shoes as an example.

2) Computer-aided design of jacquard spacer sports shoes with separated function areas is introduced according to the physical characteristics of feet and the requirement of favourable climatic regulation for sports shoes. These functional areas filled with versatile net variations can be seamlessly stitched by Piezo-Jacquard technics, giving the jacquard spacer sports shoes a fashionable look and functional performance of good air permeability, well-directed moisture and heat dissipation.

\section{Acknowledgements}

The authors acknowledge the financial support from the National Science Foundation of China (No.11302085 and 51403080), the Fundamental Research Funds for the Central Universities (No. JUSRP51404A) and the Innovation fund project of Cooperation among Industries, Universities \& Research Institutes of Jiangsu Province (No. BY2014023-34 and BY2014023-20).

\section{References}

[1] Jiang G, Feng X. A simulation model of Lace made on a multibar Raschel machine. Fibers\& Textiles in Eastern Europe, 2008, 16(2): 58-60.

[2] Zhang Y, Jiang G, Yao J, Tong Y. Intelligent segmentation of jacquard warp-knitted fabric using a multiresolution Markov random field with adaptive weighting in the wavelet domain. Textile Research Journal, January 2014, 84: 28-39.

Table 3. Jacquard combined units of separated functional areas

\begin{tabular}{|l|c|c|c|c|c|}
\hline $\begin{array}{c}\text { Functional } \\
\text { areas }\end{array}$ & A & B & C & D & \\
\hline & & & & & \\
\hline \\
Jacquard
\end{tabular}


[3] Ye X, Hu H, Feng X. Development of the Warp Knitted Spacer Fabrics for Cushion Applications. Journal of Industrial Textiles, 2008, 37: 213-223.

[4] Guo X, Long H, Sun Y, Zhao L. Theoretical modeling of spacer-yarn arrangement for warp-knitted spacer fabrics and experimental verification. Textile Research Journal, 2013, 83:1467-1476.

[5] Ye X, Fangueiro R, Hu H, Araújo M. Application of warpknitted spacer fabrics in car seats. Journal of the Textile Institute, 2007, 98: 337-344.
[6] Miao X, Ge M. The compression behaviour of warp knitted spacer fabric. Fibres\& Textiles in Eastern Europe, 2008, 16: $90-92$.

[7] Cong $H$, Ge M, Jiang G. Three-dimensional simulation of warp-knitted fabric. Fibers\& Textiles in Eastern Europe, 2008, 17(3): 66-69.

[8] Cong H, Li X, Zhang A, Gao Z. Design and Simulation of Jacquard Warp-knitted Towel Fabric. Fibers\&Textiles in Eastern Europe, 2014, 22(5): 54-58. 